Available online on 15.03.2020 at http://jddtonline.info
Open Access to Pharmaceutical and Medical Research
unrestricted non-commercial use, provided the original work is properly cited

Open@ Access

Research Article

\title{
Expressions of biomarkers in MCF7 Breast and Colon Cancer Cell Lines
}

\section{Poornima BN*, Farah Deeba}

Department of Biosciences, CMR University, Bangalore, Karnataka, India

\begin{abstract}
Cancer is one of the leading causes of death which accounts for $13 \%$ of total deaths, worldwide. Colorectal and Breast cancer fall under main categories according to World Health Organisation (WHO) cancer facts sheet ${ }^{1}$. The need to understand the expression of clinical biomarkers in breast cancer and colon cancer is necessary for diagnosis and therapeutic response. In this article, the expressions of histone H1 and TP53 biomarkers were established for four different colon cancer cell lines and compared with the expressions of MCF7 cell line. The results show varied expression of Histone H1 along with TP53 markers among the cell lines. The results suggest that the linker Histone H1 has shown nuclear localization in HCT 116p53 wt (wild type) cancer cell line whereas it has shown cytoplasmic distribution in the respective nu ll type cell line with intense expression. The result also suggests that a localized distribution in HRA19 cells and a diffused distribution in SNU-C2B cell line. These established results were compared with the expression of the biomarkers in MCF7 in order to get a better understanding.
\end{abstract}

Keywords: Tumour Proteins, p53, H1, MCF-7 cell, HCT116, HRA19.

Article Info: Received 04 Jan 2020; Review Completed 17 Feb 2020; Accepted 25 Feb 2020; Available online 15 March 2020

Cite this article as:

Poornima BN, Deeba F, Expressions of biomarkers in MCF7 Breast and Colon Cancer Cell Lines, Journal of Drug Delivery and Therapeutics. 2020; 10(2):107-114 http://dx.doi.org/10.22270/jddt.v10i2.3993

*Address for Correspondence:

Poornima BN, Department of Biosciences, CMR University, Bangalore, Karnataka, India

\section{INTRODUCTION:}

1.1 Biomarkers: A biomarker is a measurable substance which helps in depicting a normal or abnormal biological system by analysing chemical modification in biomolecules. A cancer biomolecule is a tool to measure the risk of developing cancer in any specific tissue. It helps in guiding clinical decision making while measuring the risk of progression or potency of therapy of a cancer. Generally biomarkers are classified into Predictive, Prognostic \& Diagnostic biomarkers ${ }^{2}$.

\subsection{Tumour Protein TP53 and Cancer}

TP53 is an essential protein which functions as 'tumour suppressor' in multicellular organisms. It is denoted as p53 or TP53 gene and is located on the short arm of chromosome 17 encodes TP53 protein whose chief function is to prevent genomic mutations while conserving the stability of the genome ${ }^{3}$. Most of the mutations hinder the ability of TP53 to bind to the target DNA, further on blocking the transcriptional activation, indicating a 'recessive loss of function mutation'. Apart from genomic conservation function, TP53 plays other roles as anticancer functions, apoptosis etc. Likewise people who inherit only one functional copy of the gene are at risk of developing early tumours ${ }^{4}$. The TP53 tumor suppressor protein plays a vital role in cellular proliferation.

\subsection{H1 Linker Histones}

Linker histones are located in the outer surface of the nucleosomal structure in a chromatin fibre and are known to be involved in compaction of the fibre and in regulating chromatin higher order structure ${ }^{5}$. These histones are not conserved across species and undergo post translational modifications on their tail regions. The variant forms of linker histones and their modifications have been shown to regulate developmental and differentiation programs ${ }^{6}$. Research has been going on to discover the relation between the linker histone $\mathrm{H} 1$ and cancer, establishing the findings and simultaneously generating further questions to be addressed. Many important findings have been established based on the research work in this area emphasizing the relation between cancer, linker histone $\mathrm{H} 1$ and tumour suppressor p53 gene.

\subsection{MCF7 Breast Cancer Cell Line:}

Breast cancer accounts for $23 \%$ of the total cancer cases and is a frequent malignancy which results to $14 \%$ of the cancer deaths. Michigan Cancer Foundation-7 (MCF 7) cells are used for this comparative study. They are widely used in Estrogen Receptor (ER)-positive tumor research experiments ${ }^{7}$.

\subsection{Colon Cancer Cell Lines:}

HCT 116 wt, HCT 116 p53 null, HRA19 \& SNU-C2B cell lines are chosen for this study. The study also involves Small 
interfering RNAs (siRNAs) and scrambled siRNA along with the expression of TP53 and H1 Histones among these cell lines. siRNA is able to regulate the expression of genes through a phenomenon as RNA interference (RNAi) or silencing RNA 8. Small interfering Ribonucleic Acid (siRNA), is a class of double-stranded RNA molecule, and generally consist 21 nucleotides, which can be used to "interfere" with the translation of proteins by binding to and promoting the degradation of messenger RNA (mRNA) at specific sequences ${ }^{9}$. While studying transfection activity of SiRNA, scrambled scRNA is used.

Experimentally, an attempt is being made to investigate the connection between the TP53 tumour suppressor protein and linker histone $\mathrm{H} 1$ expression in colorectal cancer cell lines to find the probable links in the cancer mechanism and diagnostics.

The aim of the work is to analyse the significance of linker histone $\mathrm{H} 1$ in colon cancer progression and the signalling of H1 through tumour suppressor gene TP53 and to compare with that of MCF7 cell lines. This work is focussed on investigating the influence of linker histone modifications in colon cancer and to compare the expression of p53 \& H1 on MCF7 \& HCT116 wild \& null type, HRA19 \& SNU-C2B colon cancer cell lines with Cell and tissue level experiments to understand the expression patterns of linker histones during the malignant and metastatic states of colon cancer. Cell culture, confocal microscopy and histopathology have been used to investigate the same. Here the focus of the research is to compare the expression among two types of cancer cell lines; MCF-7 and colorectal cell lines- HCT116 p53 wt, HCT116 p53-/-, HRA19 and SNU-C2B and colorectal cancer tissues. Cell lines of malignant colon cancer show varied levels of linker histone expression in the absence of the tumour suppressor p53 and at metastatic stages. Cancer tissues show differential expression of $\mathrm{H} 1$ and are being investigated in detail.

The experiment goes on first with the culturing of the above mentioned four cell lines and noting the expression and variations of the linker histone $\mathrm{H} 1$ expression in the respective cell lines by Immunofluorescence staining and confocal microscopy imaging. The difference in level of intensity of $\mathrm{H} 1$ expressions in the following four cell lines indicates towards the role of the effect of the different TP53 mutations in each of the respective four colorectal cancer cell lines as found for these variations. Based on this, silencing the TP53 tumour suppressor gene is attempted in the above mentioned cell lines by siRNA (small interfering or short interfering or silencing RNA) transfection followed by looking at the expression levels of $\mathrm{H} 1$. This will help validate the role of TP53 in H1 expression in the colon cancer cell lines.

\section{MATERIALS AND METHODS}

In order to investigate the linker histone $\mathrm{H} 1$ profile in colon cancer, the malignant colon cancer cell lines were used. Cell culture, Immunofluorescence and Confocal microscopy were used for the experimental analysis followed by image processing with help of Dextrose Technologies.

\subsection{Cell culture}

Malignant human colon cancer cell lines HCT116 wt (having wt p53), HCT116 p53-/- and p53 mutant cell lines HRA19 (Agr273His) and SNU-C2B (Arg273His) from ATCC. HCT116wt cell line was cultured in RPMI-1640 supplemented with $10 \% \mathrm{FBS}$ and the remaining cell lines were cultured using DMEM (Dulbecco's Modified Eagle Medium) supplemented with 10\%FBS 10.

30,000 cells from each of the cell lines were plated in $35 \mathrm{~mm}$ cover slip stuck petridishes and allowed to grow for $24 \mathrm{hrs}$ and then taken for Immunofluorescence.

Table I. Colon cancer cell lines with their TP53 genes status

\begin{tabular}{|c|c|}
\hline Cell Line & Status of p53 gene \\
\hline HCT 116 wt & p53 wt \\
\hline HCT 116 p53 null & p53 -/- \\
\hline HRA19 & p53 with Arg273His mutation \\
\hline SNU-C2B & p53 with Arg273His mutation \\
\hline
\end{tabular}

\subsection{Immunofluorescence}

After the growth period of 24hrs, immunofluresence protocol was followed for further processing. The medium was aspirated and after washing with $1 \mathrm{x}$ PBS (2-3 times), cells were fixed with $4 \%$ paraformaldehyde (10 mins). After PBS wash cells were permeabilised with $0.1 \%$ Triton X-100 in $1 \mathrm{x}$ PBS. Blocking is done with 5\% BSA in PBS for 1 hour at RT. Primay antibody against H1 (Anti-H1 (AE-4) mAb, Santa cruz Biotechnology Inc.) was added and kept in $4^{\circ} \mathrm{C}$ for overnight. After PBS wash the next day, secondary antibody (Anti-Mouse IgG - AlexaFluor 647) was added and incubated for 1 hour RT. DAPI staining was performed and incubated for 10 minutes and then the cells were taken for imaging 11 .

\section{3 p53 siRNA Transfection}

2x $10^{5}$ cells were plated /ml and kept for overnight incubation. After 24hrs, 50nm each of p53siRNA and p53 scrambled siRNA were transfected into the cells with DharmaFECT transfection media. Initially, $50 \mathrm{~nm}$ of siRNA is added to $2 \mu \mathrm{L}$ of Dharmafect is added to $100 \mu \mathrm{L}$ of optiMEM and the vials are kept for 5 mins at room temperature. Meanwhile the medium is aspirated from the plates and later $900 \mu \mathrm{L}$ of optiMEM is added to the plates. Then the siRNADharmafect transfection mixture is added to the cells and incubated for $48 \mathrm{hrs}$. After $48 \mathrm{hrs}$, cells were trypsinised and replated at a concentration of $3 \times 104$ cells $/ \mathrm{ml}$ and incubated overnight $\left(4 \% \mathrm{CO}_{2} ; 37{ }^{\circ} \mathrm{C}\right)$. The cells were then taken through the Immunofluorescence assay against H1 12. 


\section{RESULTS}

3.1 Phase I. Linker histone H1 expression in malignant colon cancer cell lines (human)

DAPI
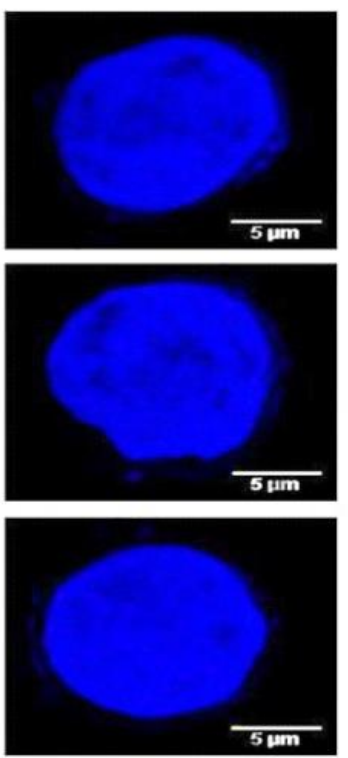

H1
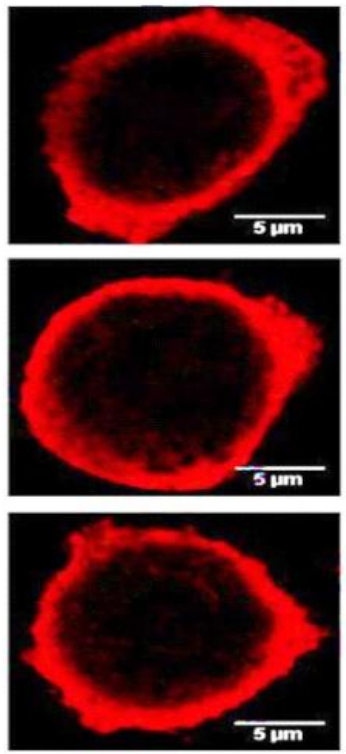

Merge
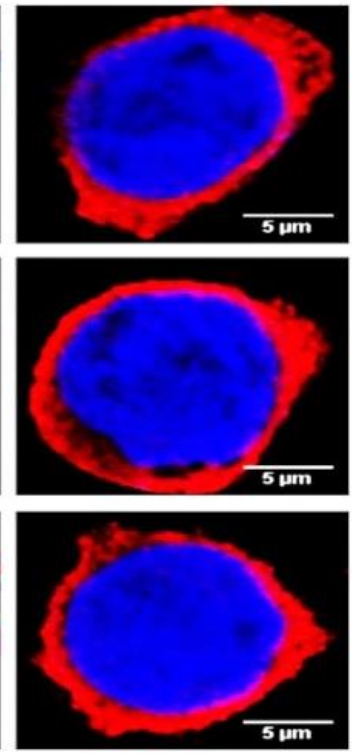

Figure I. Nuclear localization of Histone H1 in malignant HCT 116 P53 wild type colon cancer cell line

DAPI

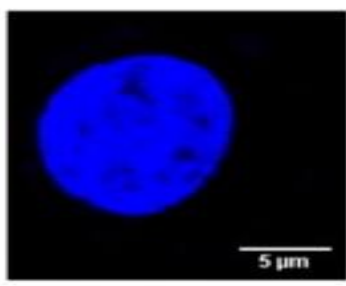

HCT 116 p53-

Cell nuclei

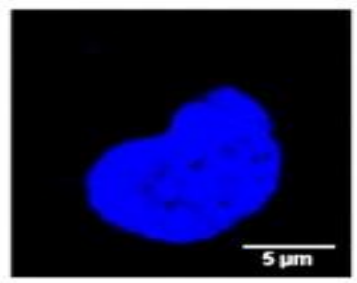

H1
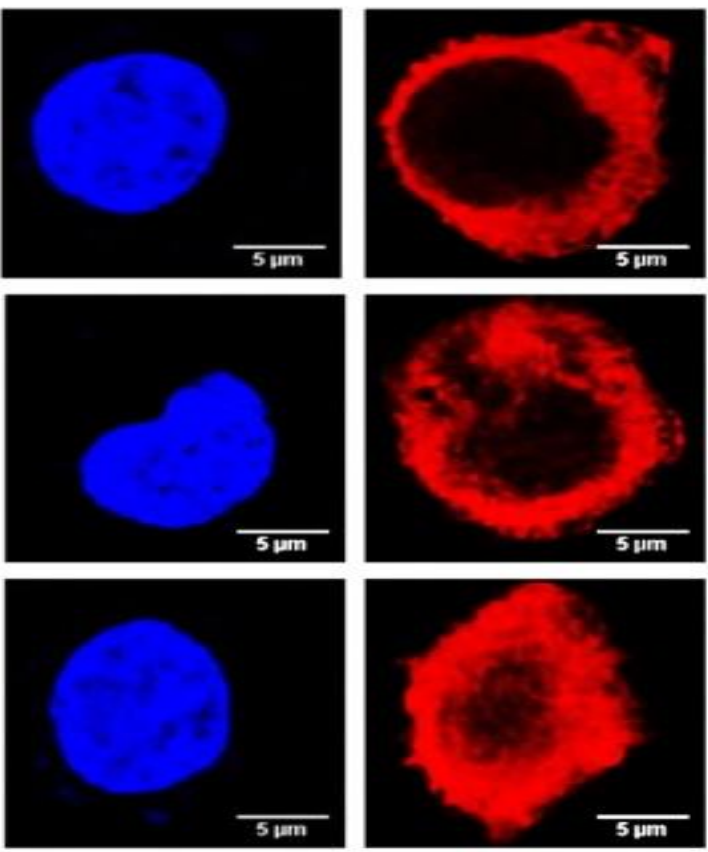

Merge
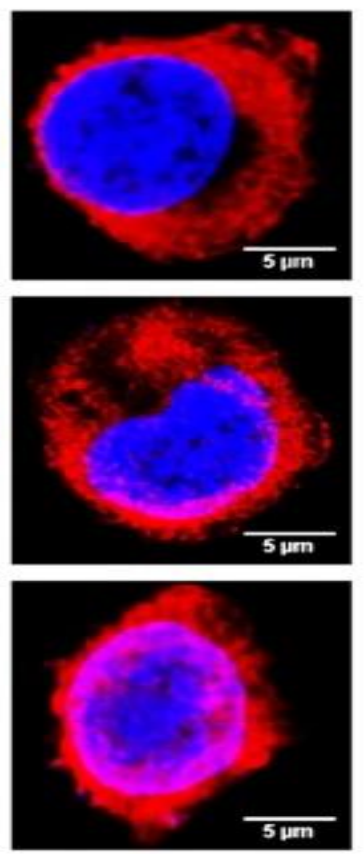

Figure II. Cytoplasmic distribution of Histone H1 in malignant HCT 116 p53 null type colon cancer cell line 


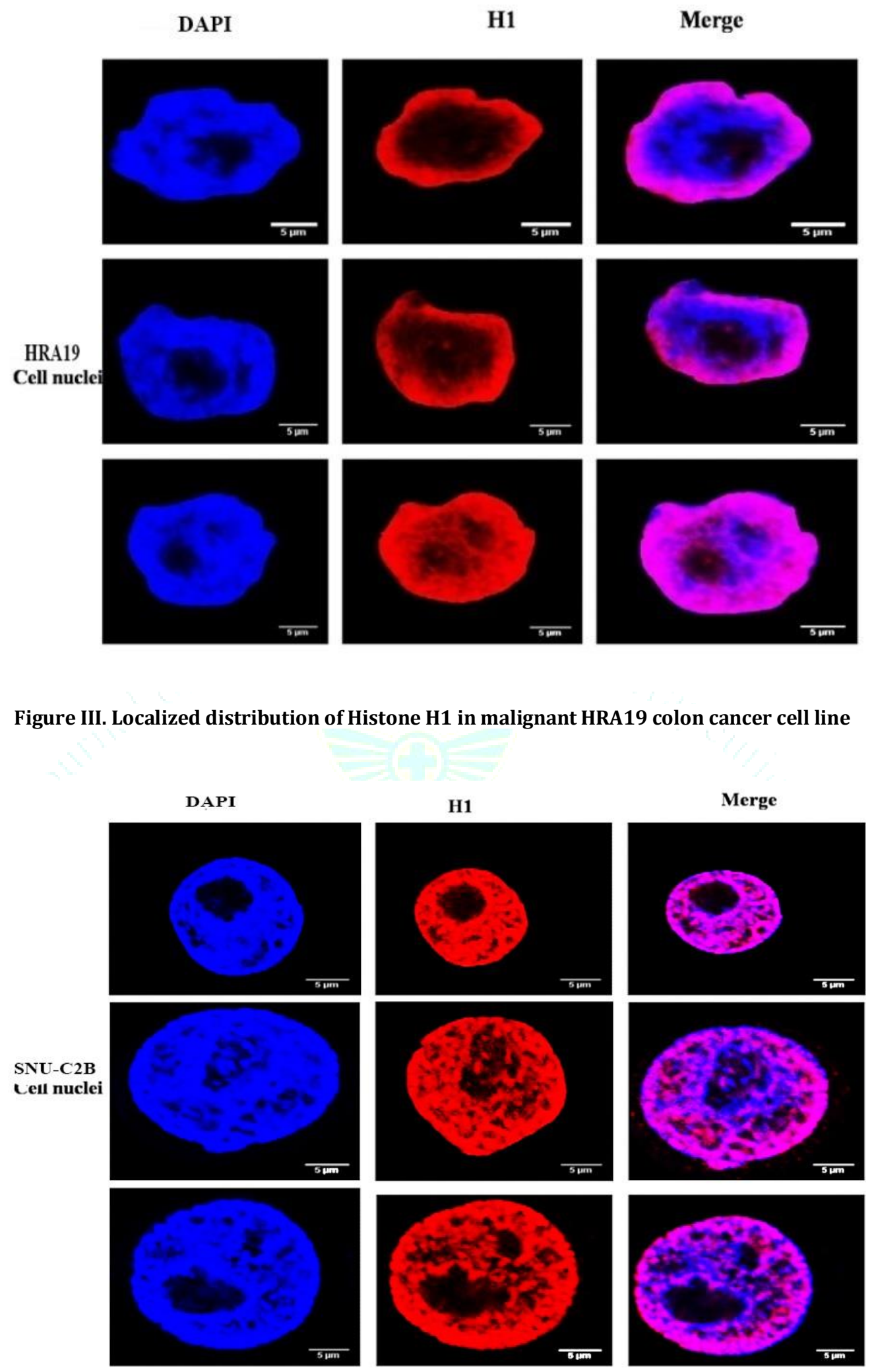

Figure IV. Diffused distribution of Histone H1 in malignant SNU-C2B colon cancer cell line 
3.2 Phase II. Linker histone expressions in view of transfection with siRNA and scrambled (sc)RNA in human colorectal cancer cell lines

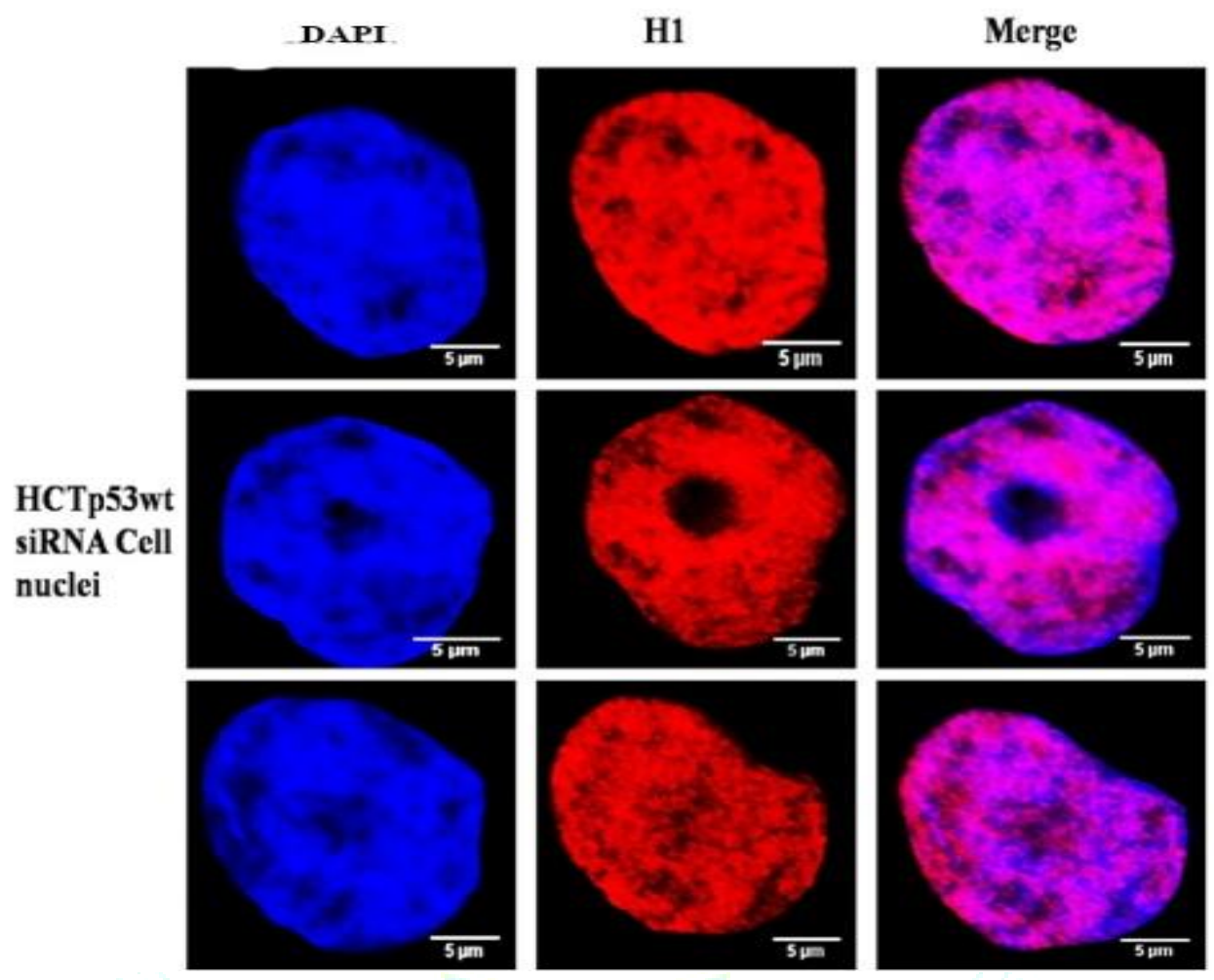

Figure V. Expression of Histone H1 in HCT116 p53 in siRNA cell nuclei.

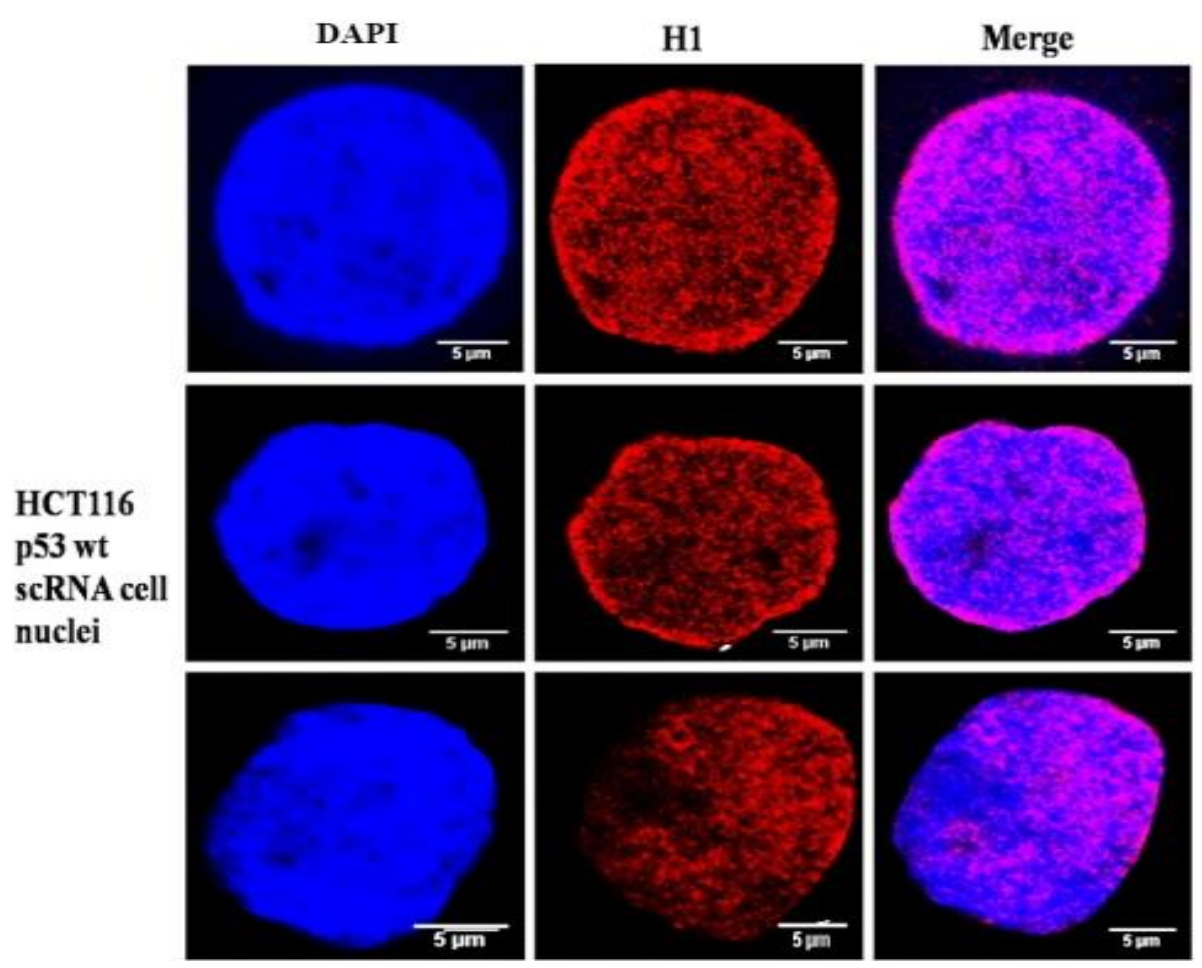

Figure VI. Expression of Histone H1 in HCT116 p53 in scRNA cell nuclei. 


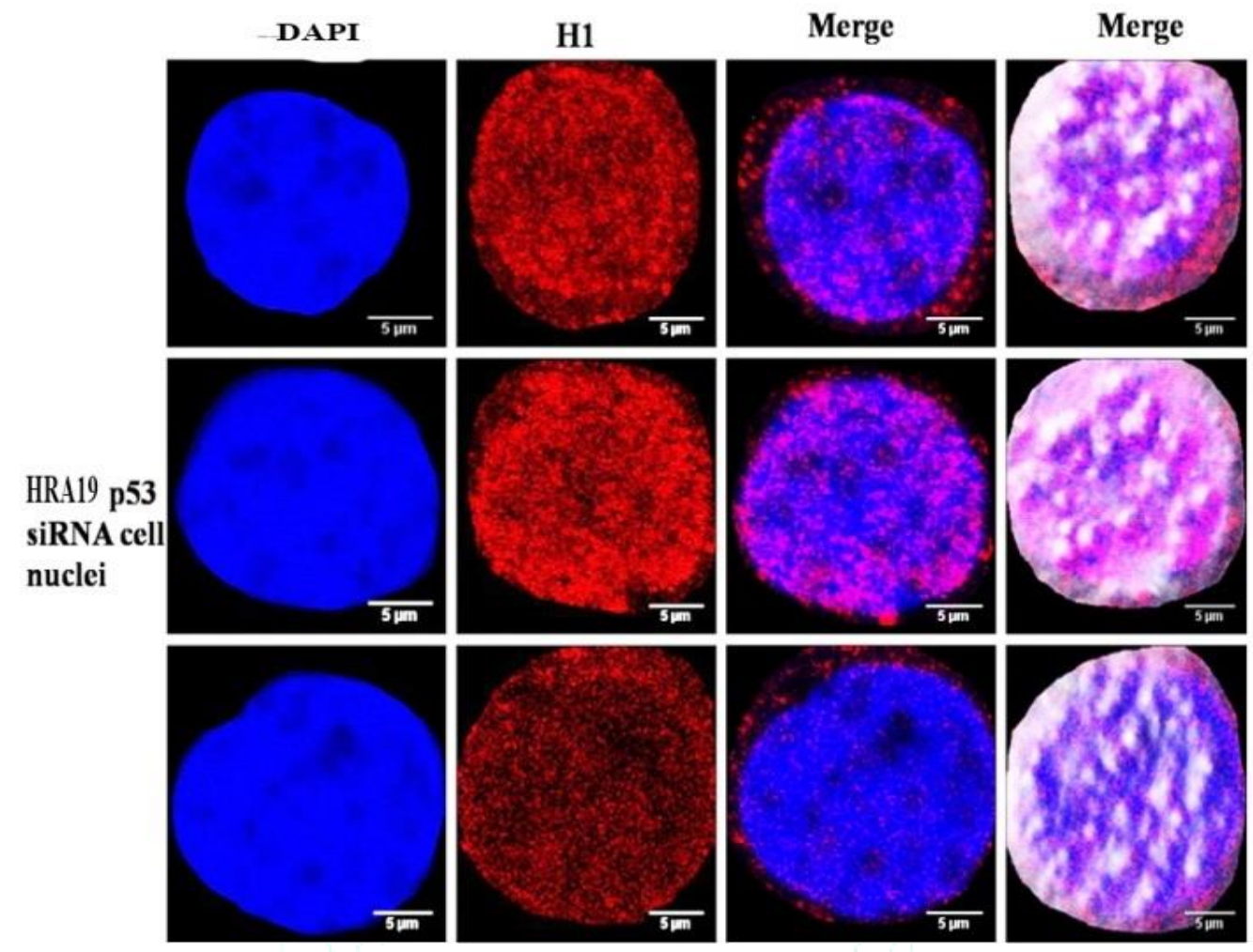

Figure VII. Expression of Histone H1 in HRA19 p53 in siRNA cell nuclei.

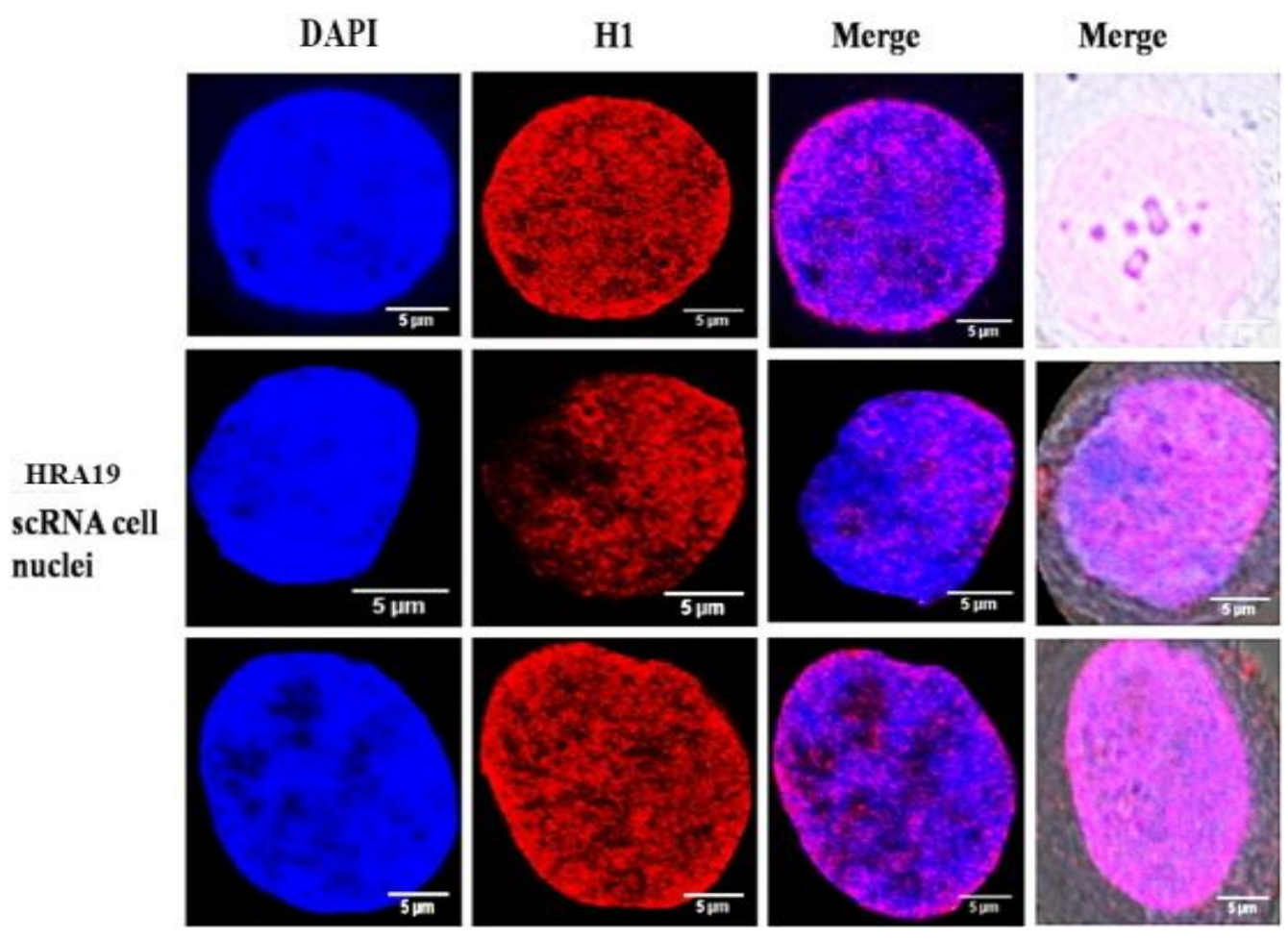

Figure VIII. Expression of Histone H1 in HRA p53 in scRNA cell nuclei. 


\section{DISCUSSIONS:}

1. From figure I, it is evident that Linker histone H1 shows nuclear localization (qued at nucleoli) in HCT 116 p53 wt colon cancer cell lines whereas HCT116 p53 -/showed more of cytoplasmic distribution (figure II), suggesting the role of $\mathrm{H} 1$ for localization. It is also obvious that the intensity of the expression is high in null type when compared with the wild type. Figure III depicts that HRA19 cancer cell lines exhibits a localized distribution while SNU-C2B reveals a diffused distribution of the linker histone $\mathrm{Hi}$ (figure IV). Intensity of histone $\mathrm{H} 1$ distribution in SNU-C2B cells is high.

2. Expression of H1 in HCT1116 wt p53 siRNA/scRNA had not showed much difference from the phase I experiments (figure I- IV) on HCT116wtp53 and HCT116p53-/- as observed in the images. HRA19 siRNA cell line has shown diffused expression of histone $\mathrm{H} 1$ to the cytoplasm, it is not in the case of HRA19 cell lines in the previous experiments with localized distribution. HRA19 scRNA has shown expression of $\mathrm{H} 1$ in cytoplasm but not as much as evident as the siRNA transfected cell lines. The intensity of expression is more in the HCT116p53wt transfected cell lines than the HRA19 cell lines.

Cell and tissue level experiments have been done to understand the expression patterns of linker histones during the malignant and metastatic states of colon cancer. Cell culture, confocal microscopy and histopathology have been used to investigate the same.

Corresponding to the above experimentation, a comparative analysis is done with MCF7 cancer cell line: various research articles are summarised as follows;

3. According to Sean W. Harshman. et.al, the neoplastic MCF-7 cell line has a greater number of histone H1.4 phosphorylations. They treated MCF7 cells with $70 \mathrm{nM}$ estradiol and conducted LC-MS to identify the changes in histone $\mathrm{H} 1$ phosphorylation. The data showed an increase in number of histone $\mathrm{H} 1$ phosphorylations detected in response to estradiol when compared to the untreated MCF-7 cells ${ }^{13}$.

4. Andrea Izquierdo-Bouldstridge et.al, suggests that expression of genes are altered as a result of Knocking down (KD) of each $\mathrm{H} 1$ variant in breast cancer cells. It also showed H1.2 and H1.4 KDs being most deleterious while exhibiting precise proliferations ${ }^{14}$.

5. Vani $G$ et.al, investigated the effects of exogenous histone $\mathrm{H} 1$ on estrogen receptor (ER) status of human breast cancer MCF 7 cells in presence and absence of estrogen. The current study revealed that treatment of exogenous histone $\mathrm{H} 1$ brings a significant decrease in the estrogen receptor which plays a vital role in chemotherapeutic treatment of breast cancer 15 .

6. Characterizing of H1.2 in MCF7 Cell is summarized as cycle arrest in G1 Phase while reduction in nucleosome spacing according to a research article penned by Xin Ye. Et.al 16.

7. A familiar tumor suppressor gene TP53 is involved in breast cancer with various mutated genes. Expression of TP53 is more frequent with higher proliferation rate in Estrogen receptors-negative (ER'). Dysfunction of TP53 is a vital incidence in breast cancer 17 .
8. Noa Rivlin et. al., put forward that most TP53 alterations is due to point mutations which yields a mal-functional proteins gets accumulated in breast tumor cells. The incidence of TP53 mutations ranges from $15-71 \%$ in breast tumours. Their work postulates that TP53 mutations at T1 stage tumours are uncommon while the frequency in T3 stage is high 4.

9. According to Torres CM, et al proposes that the histone levels of $\mathrm{H} 1$ varied significantly among various tumor cells. Glioblastoma and breast cancer cells were found out to be more hostile tumors with the highest proportion of cells while having low levels of H1.0 18 .

10. Elledge R.M, et.al., suggests that the alteration in TP53 suppressor genes is high and hence it plays a vital role in the development of breast cancer while accelerating more rapid cell proliferation on loss of TP53 suppressing activity ${ }^{19}$.

11. According to the report postulated by Massimo Negrini,et.al., it is evident that a minimum of two genetic events are necessary to convert tumor suppressor genes on chromosome 17 which aids in the development of breast cancer 20 .

12. Research work of Melissa A Troester infers that the expression of 747 genes correlates directly with p53 status in Breast cancer cell lines 21.

\section{CONCLUSIONS}

Cell lines of malignant colon cancer show varied levels of linker histone expression in the absence of the tumour suppressor TP53 (p53) and at metastatic stages. Cancer tissues show differential expression of $\mathrm{H} 1$ and are being investigated in detail. The results of these cell lines showing differential molecular responses provide a great impact on the diagnosis, prognostication, and tumor-targeted cancer therapy. Comparison of biomarkers of H1, TP53 \& siRNA signalling in MCF7 Breast cancer and HCT116, HRA \& SNUC2B colon cancer cell lines denotes tumor grades. Interactions and expressions of $\mathrm{H} 1$ play significant role in cancer therapeutics while making histone $\mathrm{H} 1$ to be a drug target

\section{REFERENCES}

1. https://www.who.int/nmh/publications/fact_sheet_cancers_en. pdf

2. Nicolas Goossens, Shigeki Nakagawa, Xiaochen Sun, Yujin Hoshida. Cancer biomarker discovery and validation. Transl Cancer Res. 2015 June; 4(3):256-269.

3. Caroline Rocha de Oliveira Lima, Rogério Elias Rabelo ,Valcinir Aloísio Scalla Vulcani, Lorena Damasio Cardoso, Nicaelle Luan de Moura Sousa, Veridiana Maria Brianezi Dignani de Moura. P53 gene: major mutations in neoplasias and anticancer gene therapy. 2012; Cienc.Rural 42 (5).

4. Noa Rivlin, Ran Brosh, Moshe Oren, and Varda Rotter Mutations in the p53 Tumor Suppressor Gene Important Milestones at the Various Steps of Tumorigenesis Genes Cancer. 2011 Apr; 2(4): 466-474.

5. Guohong Li and Danny Reinberg Chromatin higher-order structures and gene regulation. Curr Opin Genet Dev. $2011 \mathrm{Apr}$; 21(2):175-186.

6. Steven Henikoff and M. Mitchell Smith. Histone Variants and Epigenetics. Cold Spring Harb Perspect Biol. 2015 Jan; 7(1):a019364.

7. Serban Comsa, Anca Maria, Cimpean, Marius Raica. The Story of MCF-7 Breast Cancer Cell Line: 40 years of Experience in Research. Anticancer Research June 2015; 35:63147-3154 
8. Hassan Dana, Ghanbar Mahmoodi Chalbatani, Habibollah Mahmoodzadeh, Rezvan Karimloo, Omid Rezaiean, Amirreza Moradzadeh, Narges Mehmandoost, Fateme Moazzen,Ali Mazraeh, Vahid Marmari, Mohammad Ebrahimi, Mohammad Menati Rashno,Saeid Jan Abadi, and Elahe Gharagouzlo. Molecular Mechanisms and Biological Functions of siRNA. Int J Biomed Sci. 2017; 13(2):48-57.

9. Elledge, R.M., Fuqua, S.A.W., Clark, G.M. et al. The role and prognostic significance of p53 gene alterations in breast cancer. Breast Cancer Res Tr 1993; 27:95-102.

10. Seung Wook Kim, Sun-Jin Kim, Robert R. Langley, Isaiah J. Fidl. Modulation of the cancer cell transcriptome by culture media formulations and cell density. International journal of oncology; 2015: 46:2067-2075.

11. https://dm5migu4zj3pb.cloudfront.net/manuscripts/69000/69 636/JCI69636sd.pdf

12. Yong-Wei Zhang, Rochelle E. Nasto, Sandra A. Jablonski, Ilya G Serebriiskii, Rishi Surana, Joseph Murray, Michael Johnson, Rebecca B. Riggins, Robert Clarke, Erica A. Golemis, Louis M Weiner. RNA Interference Screening to Identify Proliferation Determinants in Breast Cancer Cells. Bio Protoc. 2017; 7(15). doi:10.21769/BioProtoc.2435.

13. Sean W. Harshman, Michael E. Hoover, Chengsi Huang, Owen E. Branson, Sarah B. Chaney, Carolyn M. Cheney, Thomas J. Rosol, Charles L. Shapiro, Vicki H. Wysocki, Kay Huebner, Michael A. Freitas. Histone H1 Phosphorylation in Breast Cancer. American Chemical Society. J. Proteome Res. 2014; 13:2453-2467.

14. Andrea Izquierdo-Bouldstridge, Alberto Bustillos, Carles BonetCosta, Patricia Aribau-Miralbes, Daniel Garc'ia-Gomis, Marc Dabad, Anna Esteve-Codina, Laura Pascual-Reguant, Sandra Peiro, Manel Esteller, Matthew Murtha, Llu'is Millan-Arino, Albert Jordan. Histone $\mathrm{H} 1$ depletion triggers an interferon response in cancer cells via activation of heterochromatic repeats. Nucleic Acids Research; 2017:45(20):11622-11642.

15. Vani G, Devi CS. Effect of histone H1 on estrogen receptor status of human breast cancer MCF 7 cells. Mol Cell Biochem. 2005; 272(1-2):151-5.

16. Ye X, Feng C, Gao T, Mu G, Zhu W, Yang Y. Linker Histone in Diseases. Int J Biol Sci 2017; 13(8):1008-1018.

17. Mariana Varna, Guilhem Bousquet, Louis-Francois Plassa, Philippe Bertheau, Anne Janin. TP53 Status and Response to Treatment in Breast Cancers. Review Article. Hindawi Publishing Corporation Journal of Biomedicine and Biotechnology. 2011; 284584.

18. Torres CM, Biran A, Burney MJ, Patel H, Henser-Brownhill T, Cohen AS, Li Y, Ben-Hamo R, Nye E, Spencer-Dene B,Chakravarty P, Efroni S, Matthews N, Misteli T, Meshorer E, Scaffidi P. The linker histone H1.0 generates epigenetic and functional intratumor heterogeneity. Science. 2016 Sep 30; 353(6307). pii: aaf1644.

19. Richard M. Elledge, Suzanne A. W. Fuqua, Gary M. Clark Pascal Pujol, D. Craig Allred. The role and prognostic significance of p53 gene alterations in breast cancer. Breast Cancer Res Tr 1993; 27:95.

20. Massimo Negrini, Silvia Sabbioni, Subrata Haldar, Laura Possati, Antonella Castagnoli, Alfredo Corallini, Giuseppe BarbantiBrodano, and Carlo M. Croce. Tumor and Growth Suppression of Breast Cancer Cells by Chromosome 17-associated Functions. Cancer Research; 1994; 54:ISI8-1824.

21. Melissa A Troester, Jason I Herschkowitz, Daniel S Oh, Xiaping He, Katherine A Hoadley,Claire S Barbier, and Charles M Perou Gene expression patterns associated with p53 status in breast cancer. BMC Cancer. 2006; 6:276. 